\title{
Medico Legal Documentation in a Public Hospital in India: An Analysis of 100 Cases
}

\author{
Vijay Kumar Tadia ${ }^{1}$, Paresh Shekhar Jangale ${ }^{2}$ \\ ${ }^{1}$ Senior Resident Administrator, AIIMS, New Delhi, ${ }^{2}$ MHA, TISS, Mumbai
}

\begin{abstract}
Introduction: Medico-legal audits are an integral part of hospital/clinical audits and involve examination of the hospitalrecords. The clinical audits aim towards improvement of quality of care and hence are limited to the scrutiny of patient's clinicalrecords. On the other hand, a medico-legal audit aims at the prevention of foreseeable litigations on the hospital and includes the perusal of all the hospital records.
\end{abstract}

Aim \& Objective: To carry out the audit of medico legal documentation in a public hospital.

Methodology: A retrospective record-based study was conducted in a 2000 bedded public hospital in the month of May-June 2012. 100 documented medico legal cases were considered using simple random sampling. The quantitative data was collected by using a checklist for the audit of medico legal case files. SPSS was used for the analysis of the data.

Results: Most of the MLC cases were found to fall in the middle age group of 21-35 years of age (32\%) and within that the maximum people contributing to the MLC cases was the sub- group of age between 2125 years $(16.66 \%)$. Out of the total recorded sex of the MLC cases, $65.96 \%$ were males and $34.04 \%$ were females. The highest number of cases recorded were of the RTA $(20 \%)$ followed by the accidental injury $(17 \%)$.

Discussion \& Conclusion: Alarge number of the cases were of the road traffic accidents (20\%)followed by accidental injury (17\%), so the emergency department should be kept well equipped to treat such cases. The proportion of the male patients $(65.96 \%)$ was double as compared to the female patients $(34.04 \%)$, so the human resource planning of the emergency department can be done accordingly.

Keywords: Medico legal cases, Medical Records, Medico legal record audit, Good Record Keeping

\section{Introduction}

A high standard of medical record keeping is important for safe patient care and provides information for research, audit and medicolegal purposes. ${ }^{1}$

An MLC is a case of injury/illness where the attending doctor, after eliciting history and examining

\section{Corresponding Author:}

Vijay Kumar Tadia

Senior Resident Administrator, AIIMS, New Delhi

e-mail : vijaytadia@gmail.com the patient, thinks that some investigation by law enforcement agencies is essential to establish and fix responsibility for the case in accordance with the law of the land. ${ }^{2,3}$

Documentation is regarded as an essential element in the legal system of country. ${ }^{4}$

Medico-legal audits are an integral part of hospital/clinical audits and involve examination of the hospitalrecords. The clinical audits aim towards improvement of quality of care and hence are limited to the scrutiny of patient's clinicalrecords. On the other hand, a medico-legal audit aims at the prevention of foreseeable litigations on the hospital and includes theperusal of all the hospital records..$^{5}$ 
Aim \& Objective: To carry out the audit of medico legal documentation in a public hospital.

\section{Methodology}

A retrospective record-based study was conducted in a 2000 bedded public hospital in the month of May-June, 2012. 100 documented medico legal cases were considered using simple random sampling. The quantitative data was collected by using a checklist for the audit of medico legal case files. SPSS was used for the analysis of the data.

\section{Results}

1. Recording of the age of the patient: It was been found that the age of the patient was recorded in $98 \%$ of the cases.

2. The proportion of the Medico-Legal Cases (MLCs) according to age: Most of the MLC cases were found to fall in the middle age group of 21-35 years of age $(32 \%)$ and within that the maximum people contributing to the MLC cases was the subgroup of age between 21-25 years (16.66\%).

3. The recording of the sex of the patient: The sex of the patient was found to be recorded in the $94 \%$ of the MLC cases. Out of the total recorded sex of the MLC cases, $65.96 \%$ were males and $34.04 \%$ were females.

4. Cause of the medico-legal cases: It was observed that diagnosis was written on all the case files $(100 \%)$. The highest number of cases recorded were of the RTA (20\%) followed by the accidental injury (17\%), a considerable amount of MLCs were recorded when a patient was shifted from a private hospital to the public hospitals (12\%).

5. The Length of stay of a MLC patient: Most of the MLC patients stayed for 1 to 5 days in the hospital (48\%) followed by stay between 6 to 10 days (23\%). The Average Length of Stay (ALOS) of the MLC patient in the hospital was 11 days.

6. Recording of the time of the injury: The time at which injury happened was recorded in $48 \%$ of the cases, it was not recorded in $38 \%$ of the cases. The question was not applicable for $14 \%$ cases.

7. Recording of the time at which patient reported to the hospital: The time at which the patient reported to the hospital was recorded in $99 \%$ of the cases and the record was found to be missing in 1 case.
8. Record of the person accompanying the patient to the casualty: In majority of the cases, the details of how the patient was brought to the casualty and who was accompanying him/her were not recorded(55\%).In the cases where such information was recorded(45), the $93 \%$ of the cases were accompanied by relatives and the rest $7 \%$ were accompanied by the police.

9. Record of the mental status: The mental status of the MLC patient was recorded in $46 \%$ of the cases and was found missing in rest of the cases (54\%).

The majority of the patients who presented to the casualty were conscious $(74 \%)$, who were in a condition to give the statement at the time of admission while there is were also considerable number of the patients who were not in the condition to give the statement(Semi-Conscious $6.5 \%$ \& Unconscious $19.5 \%)$.

10. Record of physical injury: In $70 \%$ of the cases, some form of physical injury was present. In considerable number of the cases there was absence of description of injury with respect to its Type (18.57\%), Dimensions (68.58\%) and Diagrammatic Representation (44.29\%).

11. The proportion of the MLC cases discharged against medical advice (DAMA): It was found that $11 \%$ of MLC patients were discharged against medical advice (DAMA) but the reasons for the same were not recorded even in one case.

12. Record of the Occupation of the MLC: The occupation of the MLC patients were recorded in only $3 \%$ of the cases.

\section{Record of the Complaints, History, Pulse and Blood Pressure}

In large number of MLCs, the documents were lacking the recording of the pulse (49\%) and the Blood pressure $(64 \%)$ of the patient. The presenting complaints were not recorded in $2 \%$ cases and history was not written in $3 \%$ of the cases.

\section{Discussion \& Conclusion}

It was found that in many cases the relevant information was found missing or was not recorded while documenting the medico legal case. The court of justice can ask for any data at any point and can even punish the hospital for not maintaining the records of the patient properly. Medical records are acceptable as per 
Section 3 of the Indian Evidence Act, 1872 amended in 1961 in a court of law. These are considered as useful evidence by the courts of law as it is accepted that documentation of facts during the course of treatment of a patient is genuine and unbiased. ${ }^{6}$

A large number of the cases were of the road traffic accidents (20\%)followed by accidental injury (17\%), so the emergency department should be kept well equipped to treat such cases. The proportion of the male patients $(65.96 \%)$ was double as compared to the female patients (34.04\%), so the human resource planning of the emergency department can be done accordingly.

The time at which injury happened was recorded in $48 \%$ of the cases. As time at which an injury or an event happens is of great medico legal importance, the recording should be made mandatory for the medical officers.

A considerable number of the patients were not in the condition to give the statement at time of admission (26\%), the CMOs should visit them again to elicit history.

A study by Bart S Selden et al considered the history, physical examination, vital signs, mental status, mental impairment and patient refusing care by paramedics in out hospitals. ${ }^{7}$

Sangeeta Regge et al in a study concluded that due to ambiguity in the understanding the MLC, more and more health care professionals are looking at MLC as a burden and hence leading to practice of defensive medicine. Their study suggested that there was a need of standard operating procedures in the context of doctors, nurses and police and their respective medicolegal roles. ${ }^{8}$

An audit of operative notes revealed that several operative notes were found incomplete $(51.57 \%)$ missing important information as CMB code (13.68\%), patient details $(6.8 \%)$ preoperative diagnosis $(6.31 \%)$, operation title $(6.31 \%)$ and postoperative instruction (14.73\%). Overall, only 92 notes were complete. ${ }^{9}$

A study on good medical record keeping revealed that the Standards measured increased over the 3 audit cycles with 3 of the indicators (writing a date, patient name and hospital number) reached $100 \%$. These results indicate medical note keeping has scope for improvement and auditing can improve standards. ${ }^{10}$

It maybe easy to state that medico-legal audit has its genesis in 'defensive medicine', but in the era of patient's awareness and judicial litigations, it must be integrated to the functioning of the health care systems. ${ }^{5}$

Ethical Clearance: Taken from institute committee

\section{Source of Funding: Nil}

\section{Conflict of Interest: Nil}

\section{References:}

1. Chamisa I, Zulu BM. Setting the records straight-a prospective audit of the quality of case notes in a surgical department - PubMed [Internet]. [cited 2020 Sep 6]. Available from: https://pubmed.ncbi. nlm.nih.gov/17892187/

2. Meera T. Medicolegal cases: What every doctor should know. JMS - J Med Soc. 2016;30(3):133-4.

3. Medicolegal issues : Guidelines to Medical Officers [Internet]. [cited 2020 Sep 5]. Available from: http://qi.nhsrcindia.org/sites/default/files/medico_ legal.pdf

4. Gutheil TG. Fundamentals of medical record documentation. Psychiatry (Edgmont) [Internet]. 2004;1(3):26-8. Available from: http://www. ncbi.nlm.nih.gov/pubmed/21191523\%0Ahttp:// www.pubmedcentral.nih.gov/articlerender. fcgi?artid=PMC3010959

5. Ateriya N, Saraf A, Meshram V, Setia P, Kanchan T, Shekhawat RS. Medico-legal Audit: An essential yet ignored aspect of healthcare in India. J Indian Acad Forensic Med. 2019;41(1):53-6.

6. Thomas J. Medical records and issues in negligence. In: Indian Journal of Urology [Internet]. Wolters Kluwer -- Medknow Publications; 2009 [cited 2020 Sep 6]. p. 384-8. Available from:/pmc/articles/ PMC2779965/?report=abstract

7. Selden BS, Schnitzer PG, Nolan FX. Medicolegal documentation of prehospital triage. Ann Emerg Med. 1990;19(5):547-51.

8. Rege S. Medico-legal cases across various hospitals - A review \& understanding of procedures. MedicoLegal Updat. 2011;11(2):71-3.

9. Lefter LP, Walker SR, Dewhurst F, Turner RWL. An audit of operative notes: Facts and ways to improve. ANZ J Surg. 2008;78(9):800-2.

10. Maiedha R. Good Medical Record Keeping. Int J Collab Res Intern Med Public Heal. 2012;4(5):53543. 